\section{Giving time back to the dentist}

Flynotes has listened to its customers' valuable feedback over the past year, and has digitalised your patient journey. So, what's new?

Treatment plans. A patient's procedures can now be grouped together into a fully accessible, bespoke and digital treatment plan for both clinician and patient to review.

Additionally, clinicians are now able to select and open a specific procedure within the treatment plan and send an email invitation to the patient to conduct an online consent review.

Patient interaction. Patients can effortlessly engage with the new Flynotes treatment consent process in the comfort of their own home, enabling informed bespoke consent to be more time-efficient for the clinician, whilst allowing the patient to understand and ask questions on a simple-to-use, fully digital and interactive treatment consent process.
Messages will be sent from the patient to the practice, building an audit trail of the treatment consent journey and understanding. Flynotes gives time back to the dentist and increases the understanding to the patient: all whilst being the gold standard in treatment consent.

Flynotes is offering a free trial until September 2020. Contact Flynotes today to get your patient consent journey fully digitalised: hello@flynotes.co.uk.

\title{
A shield that captures aerosols during drilling and scaling
}

Splashes and aerosols produced by dental instruments like ultrasonic scalers and high-speed handpieces harbour potentially dangerous pathogens. Studies show that viruses trapped within these aerosols can travel up to 20 feet from an infected person before settling on operatory surfaces and lingering in the air for hours - even days - following patient care. So, the best way to protect everyone in the operatory is to capture and contain them.

CareShield is an innovative shield that captures aerosols created during procedures such as drilling or ultrasonic scaling. Developed by Orsing, a division of Directa Dental Group, the system consists of a coneshaped, autoclavable connector that attaches to a disposable clear shield, which is changed between each patient. The CareShield system connects to the dental delivery unit for high-volume suction.

CareShield can be used in conjunction with Hygoformic by Orsing, a mouldable saliva ejector that adjusts its shape and size to fit each unique patient. Hygoformic can be used with either low- or high volume suction and is designed using a bioplastic material made from sugarcane making it a very ecofriendly solution.

For more information about CareShield contact orsing@orsing.se.

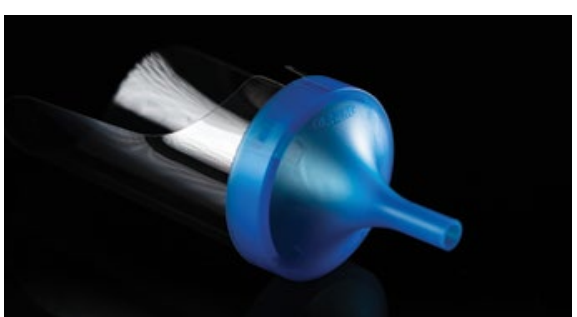

\section{At-home aligner service launched}

Dentist Dr Richard Marques has collaborated with Diamond Whites to launch an at-home aligner service which is $70 \%$ cheaper than traditional braces. Customers receive full support from $\mathrm{Dr}$ Marques, who is based in Harley Street, London, throughout their treatment. The treatment works in three simple steps:

1. Impressions are taken using the Home Impression kit and then returned, or by visiting one of the many scan centres where qualified dental staff will assess impressions for suitability

2. Once the dentist has approved the impression and created the unique plan, patients are sent a before and after 3D visual for approval

3. Once they have approved the 3D plan and made payment (either in a single payment or using finance options) Diamond Whites deliver all of the aligners. This whole process is done in under two weeks. On average customers complete their transformation in six months.

Diamond Whites Invisible Aligners are $£ 1,400$. This can be paid in full or by using the interest free finance option from $£ 1.20$ a day (three years but this can be reduced or increased to four). For more information visit https://dwaligners.co.uk/.

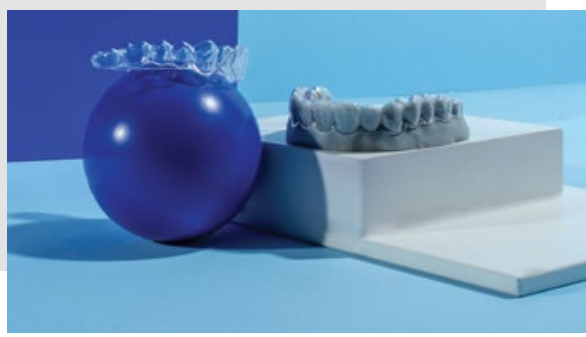

